\title{
Microwaves holography revealed by photothermal films and lock-in IR thermography: Application to NDE of dielectric and radar absorbing materials
}

\author{
by D.L. BALAGEAS, P. LEVESQUE, M. NACITAS, \\ J.-C. KRAPEZ, G. GARDETTE \\ L3C (Sensors, Characterization \& NDE Lab.), ONERA, BP 72, 92322 Châtillon cedex, France
}

\begin{abstract}
The amplitude and phase space distributions of EM (electromagnetic) fields ( $X$ - and Ku bands) are imaged and measured using microwaves interferometry revealed by photothermal films and lock-in infrared thermography. Such EM fields imaging is a powerful tool for NDE (non Destructive Evaluation) of dielectric and radar absorbing materials.
\end{abstract}

\section{Nomenclature}

E electrical field

$\mathrm{H}$ magnetic field

$P$ dissipated volume power

$\mathrm{T}$ increase of temperature

$\begin{array}{ll}\text { Greek symbols } \\ \mathrm{x}, \mathrm{y}, \mathrm{z} & \text { space coordinates } \\ \varepsilon & \text { permittivity } \\ \varphi & \text { phase } \\ \mu & \text { permeability } \\ \omega & \text { angular frequency }\end{array}$

\author{
$\sigma \quad$ electrical conductivity \\ $\xi \quad$ film sensitivity \\ Subscripts \\ 1,2 related to antenna \#1,2
}

\section{Principle of photothermal analysis of em fields intensity}

The absorption of an electromagnetic (EM) field $\mathrm{E}, \mathrm{H}$, by materials of complex permittivity, $\varepsilon=\varepsilon^{\prime \prime}-\mathrm{j} \varepsilon^{\prime \prime}$, complex permeability, $\mu=\mu^{\prime}-j \mu^{\prime \prime}$, and real part of the conductivity, $\sigma^{\prime}$, produces heat with a dissipated volume power given by the well known formula:

$$
P_{\text {abs }}=1 / 2\left[\left(\sigma^{\prime}+\omega \varepsilon^{\prime \prime}\right) E^{2}+\omega \mu^{\prime \prime} H^{2}\right],
$$

where $\mathrm{E}$ and $\mathrm{H}$ are the modulus of the electric and magnetic fields. The resulting heating can be detected by IR thermography (technique called EMIR, for ElectroMagnetic InfraRed). It is controlled by the materials thermal properties and by heat transfer to the surroundings. The analysis of the space distribution and time evolution of the temperature increase allows to identify the heat sources, $P$ abs $(x, y, z)$, which are controlled by the strength, space distribution and orientation of the EM field, and by the EM properties of the material, which acts as an array of photothermal sensors. If these properties of the material are known, identification of these distributed heat sources can finally lead to the knowledge of the intensity distribution of the incident EM field. The identification is optimized when the photothermal transducer is a thin film.

Such a technique is a close mixing of electromagnetism and thermics. The use of both thin resistive photothermal films and lock-in IR thermography allows to image, in a quantitative way, the intensity of EM fields, with a dynamic range sufficiently extended for EM purposes. Reference [1] describes the optimisation of these films and the performances of the technique: space and time modulation transfer functions and sensitivity to the electric field.

\section{Need of a phase measurement}

The main limitation of the technique was essentially due to the fact that the photon-heat conversion only allows to measure field intensity (square of the amplitude). Such a measurement impedes to reconstruct the full field, since the phase remains unknown. It is obvious that an EMIR method allowing phase measurements would be a real progress, especially for nearfield characterization of antennas. It is the reason why phase measurement was needed. Guided by optical techniques which very often use interferometric arrangements, the idea was to produce microwaves interferences for several phase values and to reveal them by photothermal films. By combining several intensity measurements it is possible to deduce both amplitude and relative phase distributions. Here the method will be very shortly presented, since we have recently presented it, with more details, in ref. $[2,3]$. 


\section{Principle of the interferometric method}

The schematic configuration is given in Fig.1 (right). The thermographic system is the one used in ref. [1] and described in ref. [4]. We either used a long-wave $(8-12 \mu \mathrm{m})$ mono detector camera (Agema 782), or an Amber focal plane array camera, working in the 3-5 $\mu \mathrm{m}$ range. The IA camera is monitoring a thin resistive photothermal film placed in a volume where two coherent EM fields are interfering. These fields can be generated by two antennas or by one antenna and an EM mirror. The phase lag between the two fields is controlled by a phase lag generator in the first configuration and by the displacement of the mirror in the second one. Furthermore, the amplitude of the EM fields are modulated in view to use the lock-in detection. In the simpler configuration the photothermal film is held perpendicularly to the common axis of the two fields, at abscissa $z=z_{0}$.

Let us consider the interferometric arrangement using two antennas (see fig. 1 right), with no sample under test in the space between antennas. Assuming, for simplification, that the electric fields of the antennas are chosen perpendicular to the direction of propagation and that they are vertically polarized, the electric field generated, at $z_{0}$, by each of the two antennas $(i=1,2)$ is:

$$
\overrightarrow{\mathrm{E}}_{1}(\mathrm{x}, \mathrm{y})=\mathrm{E}_{1}(\mathrm{x}, \mathrm{y}) \cdot e^{-\mathrm{j} \varphi_{1}(\mathrm{x}, \mathrm{y})} \cdot \overrightarrow{\mathrm{u}}_{\mathrm{y}} ; \overrightarrow{\mathrm{E}}_{2}(\mathrm{x}, \mathrm{y})=\mathrm{E}_{2}(\mathrm{x}, \mathrm{y}) \cdot e^{\mathrm{j} \varphi_{2}(\mathrm{x}, \mathrm{y})} \cdot \overrightarrow{\mathrm{u}}_{\mathrm{y}}
$$

At this point, the temperature increase $T(x, y)$ of the photothermal film (film sensitivity $\xi$ ), is proportional to the intensity of the vectorial sum of the two incident fields:

$$
\begin{aligned}
\mathrm{T}(\mathrm{x}, \mathrm{y}) & =\xi\left|\overrightarrow{\mathrm{E}}_{1}(\mathrm{x}, \mathrm{y})+\overrightarrow{\mathrm{E}}_{2}(\mathrm{x}, \mathrm{y})\right|^{2}=\xi\left|\overrightarrow{\mathrm{E}}_{1}(\mathrm{x}, \mathrm{y})\right|^{2}+\xi\left|\overrightarrow{\mathrm{E}}_{2}(\mathrm{x}, \mathrm{y})\right|^{2} \\
& +2 \xi\left|\overrightarrow{\mathrm{E}}_{1}(\mathrm{x}, \mathrm{y})\right| \cdot\left|\overrightarrow{\mathrm{E}}_{2}(\mathrm{x}, \mathrm{y})\right| \cdot \cos \left(\varphi_{2}(\mathrm{x}, \mathrm{y})-\varphi_{1}(\mathrm{x}, \mathrm{y})\right)
\end{aligned}
$$

The resulting temperature increase is then a function of the individual temperature increases caused by each antenna $(i=1,2)$, which are independent of phase, $T_{i}(x, y)=\xi\left|\vec{E}_{i}(x, y)\right|^{2}$, and of the phase lag: $\Delta \varphi=\varphi_{2}(x, y)-\varphi_{1}(x, y)$. By combining three successive measurements: $T_{1}(x, y)$ and $\mathrm{T}_{2}(\mathrm{x}, \mathrm{y})$ obtained respectively when antenna \#1 and \#2 are operating alone, and $T(x, y)$ with both antennas, the phase lag $\Delta \varphi$ can be deduced:

$$
\Delta \varphi=\operatorname{arcos}\left(\frac{T(x, y)-T_{1}(x, y)-T_{2}(x, y)}{2 \sqrt{T_{1}(x, y) \cdot T_{2}(x, y)}}\right)
$$

In order to determine the phase sign, a fourth measurement is performed, $T^{\prime}(x, y)$, with both antennas active while introducing an extra phase lag of $\pi / 2$. From the new trio $\left(T_{1}(x, y), T_{2}(x, y)\right.$ and $T^{\prime}(x, y)$ ) the sine of the phase lag $\Delta \varphi$ is deduced and it is then possible to know in which quadrant the angle $\varphi$ is situated.

Similar procedure and formulas are obtained in the second possible interferometric arrangement (one antenna and an electromagnetic mirror) - see ref. [2].

\section{Application to non destructive testing}

It is possible to achieve NDE of EM materials by measuring the EM field in their close vicinity, using EMIR method [5]. In effect, the EM field is distorted by the sample and, locally, by the possible defects. Until now, this field analysis was achieved with EMIR method by just imaging the amplitude of the reflected or transmitted EM fields (see fig. 1 left). The interferometric method and the phase analysis presented here give new possibilities for defect detection and characterization.

Two examples will be given to illustrate the advantages of the new method. They were obtained with the Agema 782, with the lock-in system from Onera/Cedip [3]. We use ellipsoidal antennas whose near field was characterized by the present interferometric method, but using both Agema 782 and Amber cameras [1]. Figures 3 and 4, taken from [1], present the radiation patterns obtained, showing the need of amplitude modulation and lock-in detection for correct measurements and the best quality of the starring array camera (dynamic range higher than $30 \mathrm{~dB}$ ). In the present NDE interferometric applications, we present just preliminary results using only the Agema camera. The part of the EM field used for sample inspection will be limited to the central isophase region ( $10 \mathrm{~cm}$ in dia.) where intensity variations are within $3 \mathrm{~dB}$. 
The first example deals with a $50 \mathrm{~mm}$-thick low absorptivity foam sample, stuck on a $5 \mathrm{~mm}$ thick PVC layer. The foam contains, at a depth of $25 \mathrm{~mm}$, a $20 \mathrm{~mm}$-dia. metallic disc (fig. 2 right). The sample is placed between the two antennas (fig. 1 right). The film is positioned at $6 \mathrm{~mm}$ from the sample. The EM frequency is $10 \mathrm{GHz}$. The transmission method (just the antenna \#2 is active) and the interferometric one (two antennas active) are applied. Results are given in fig. 5. In transmission mode, the diffraction pattern due to the insert is clearly visible, but rather intricate. For a diagnostic on the shape and size of the defect, it is necessary to make theoretical calculations and compare them to the experiment. With interferometry, the thermal images show with a better accuracy the location, shape and size of the embedded defect (see intensity and phase distributions in fig. 6) and it is possible to adjust the phase lag to enhance the contrast due to the defect.

The second example concerns a radar absorbing material containing an artificial defect also made of a different absorber (see fig. 2 left). Figure 7 presents the EMIR images obtained with the same set up used in the first example, in transmission and interferometric modes. In spite of the intricate pattern of the field due to edge effects, the presence of the internal non homogeneity is more clearly revealed by interferometry, especially in the phase image. The location and size of the defect is given with a better spatial resolution. Concerning the edge effect patterns, the subtraction of images of a sound reference sample could help in the interpretation of the images of the sample containing defects. Figure 8 gives amplitude and phase distributions along the horizontal central axis of the interferometric images of fig. 7 . They are compared to steady-state (no amplitude modulation of the EM field) interferometric results. The interest of amplitude modulation and lock-in detection clearly appears. This technique greatly enhances spatial resolution. This is particularly effective for the phase. It also avoids distortions due to convection, which is not well visible here, since fig. 8 presents only horizontal distributions. The interesting fact is the better signal to noise ratio in the phase image, if we consider the edge effect as a noise. In effect, the peak corresponding to the defect is near of $120^{\circ}$ in phase and the edge effect only $40^{\circ}$, which correspond to a ratio of 3 . This pattern is similar in the amplitude distribution, but weaker (the ratio is of 2 ).

From these two examples of NDE application of interferometry, we can state that: i) interferometry leads to better results than transmission method which only provides the field intensity; ii) phase images give information on the defects with a better spatial resolution and better signal to noise ratio than amplitude images.

\section{Conclusion}

The EMIR technique until now had the limitation of just measuring the intensity of EM fields. In most of the problems interesting microwaves engineering the lack of information on the phase was redhibitory. The interferometric method overcomes this weakness. The unknown field is interfering with an other field which is known, generated by a reference antenna or by reflection of this unknown field onto an EM mirror. Several intensity measurements of the interfering fields, using EMIR method, lead to the direct mapping of the phase of the unknown field. The method can be applied to NDE problems. The use of phase maps instead of amplitude maps in NDE provides a better spatial resolution and a higher contrast for defect detection.

\section{REFERENCES}

[1] BALAGEAS (D.L.) , LEVESQUE (P.), NACITAS (M.), KRAPEZ (J.-C.) and GARDETTE (G.) Photothermal mapping of electromagnetic fields: state-of-the-art. Proc. of the IXth Int. Conf. on Photoacoustic and Photothermal Phenomena, Nanjing (China), 26-29 June 1996, to be published as a suppl. of Progress in Material Sc. (China).

[2] BALAGEAS (D.L.), LEVESQUE (P.), and NACITAS (M.) - Microwaves holography revealed by photo-thermal films and lock-in IR thermography. Ibidem

[3] NACITAS (M.) and LEVESQUE (P.) - Mesure d'un champ électromagnétique complexe par interférométrie microonde associée à la thermographie infrarouge. $g^{\mathrm{e}}$ Journ. Nat. Microondes, Paris, April 1995.

[4] BALAGEAS (D.L.), LEVESQUE (P.), and DÉOM (A.) - Characterization of electromagnetic fields using lock-in IR thermography. Thermosense XV, SPIE vol. 1933, pp. 274-285, 1993.

[5] LEVESQUE (P.), DÉOM (A.), and BALAGEAS (D.L.) - NDE of absorbing materials using microwave stimulated infrared thermography, in QIRT'92, ed. D. Balageas, G. Busse, G.M. Carlomagno, Editions Europ. Thermique et Industrie, Paris. 92, pp. 302-307, 1992. 

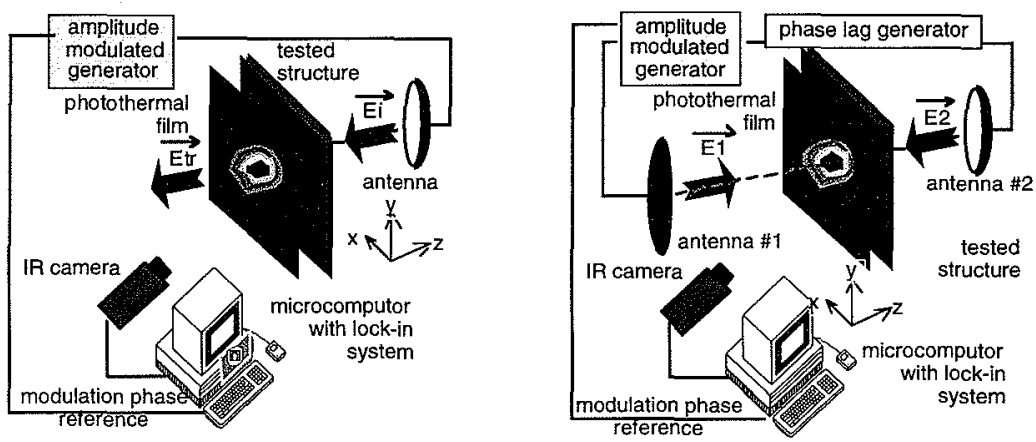

Fig. 1 - Schematic configurations for NDE by EMIR methods. Left: classical EMIR arrangement with a unique antenna - Right: Interferometric EMIR arrangement, here with two antennas.
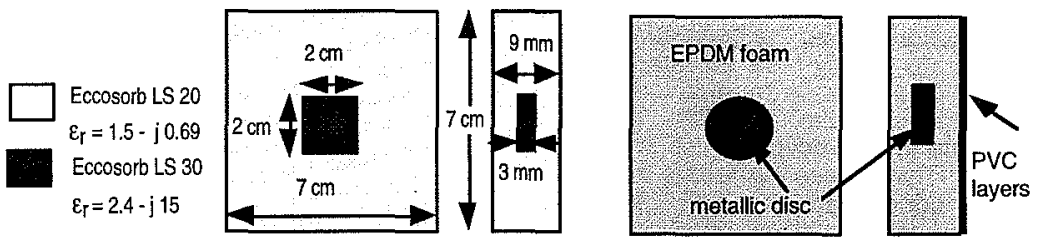

Fig. 2- Samples with artificial defects. Left: radar absorbing material; right: EM transparent material
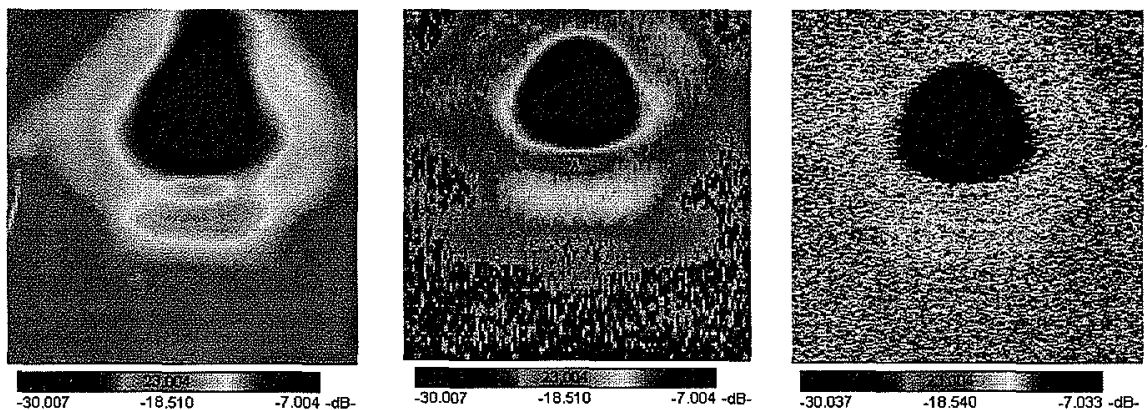

Fig. 3 - Near field radiation pattern of an ellipsoidal antenna, at $12 \mathrm{GHz}$. From left to right: i) steady state measurement with the Amber camera (400 frames added); ii) lock-in detection at $1 \mathrm{~Hz}$, with the Amber camera (492 frames added); iii) idem with the Agema 782 LW (4000 frames added).
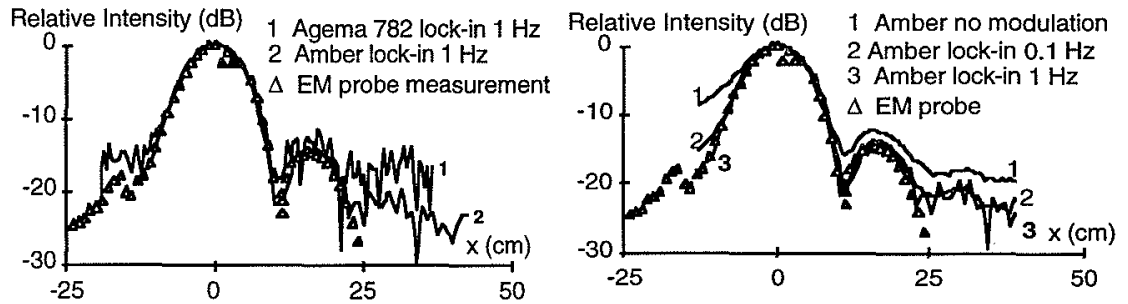

Fig. 4 - E-plane distribution of electric field, from IR images of fig. 3 and from an EM probe measurement. Left: Agema and Amber cameras at $1 \mathrm{~Hz}$; right: results of Amber camera at $0,0.1$, and $1 \mathrm{~Hz}$ 


\section{http://dx.doi.org/10.21611/qirt.1996.012}
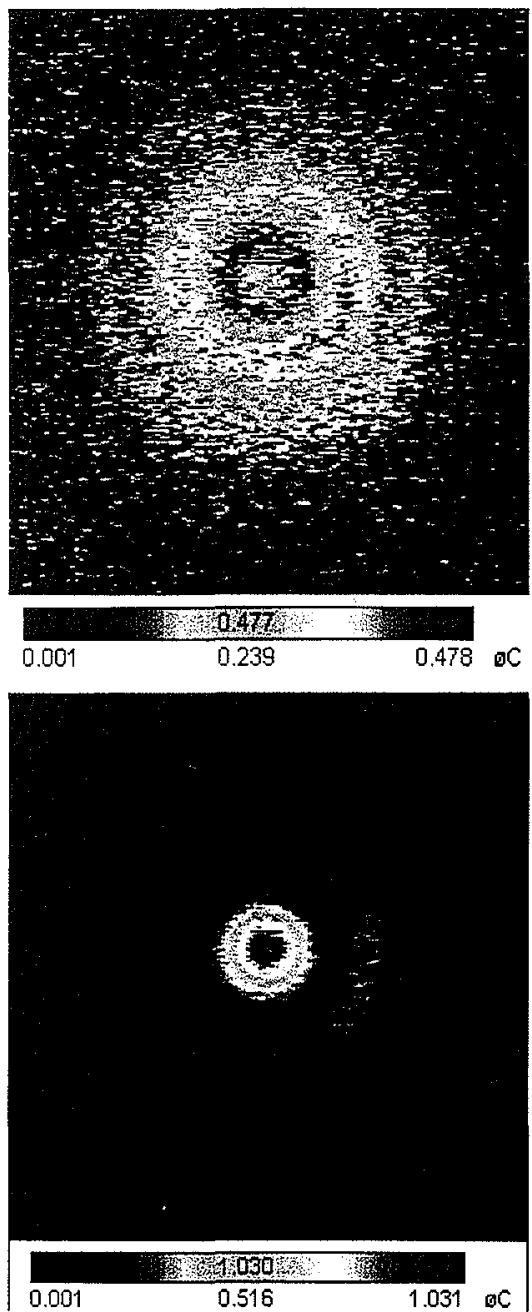

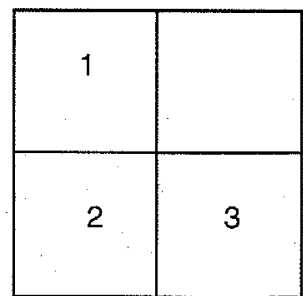

$1:$ Intensity from transmission measurement 2: Intensity from interferometry 3: Phase from interferometry

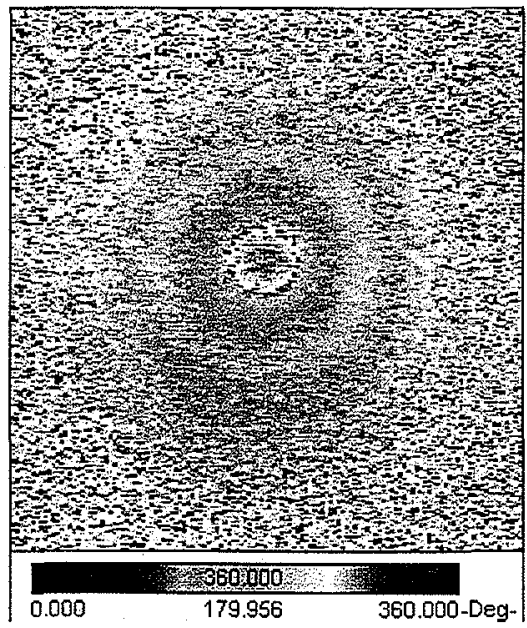

Fig. 5 - EMIR NDE of the low absorption sample of fig.2 (right). Amplitude modulation and lock-in detection at $1 \mathrm{~Hz}$ with an Agema 782. Comparison between transmission and interferometric methods.
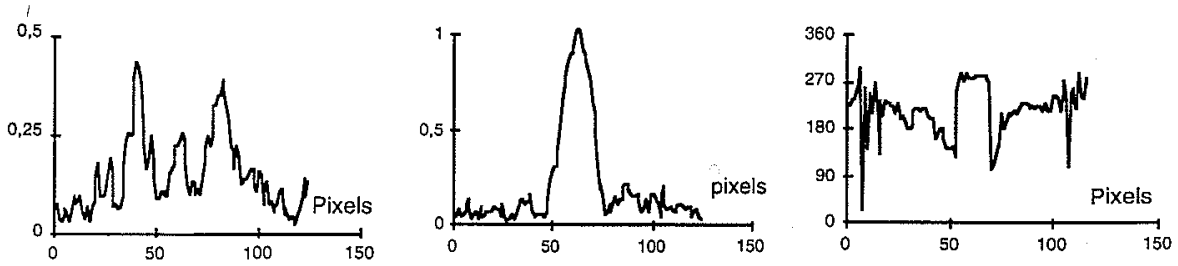

Fig. 6 - Horizontal distributions of amplitude and phase from the images of fig. 5. From left to right: i) amplitude from transmission measurement, ii) and iii) amplitude and phase by interferometric method. 
Intensity $(\mathrm{V} / \mathrm{m})^{2}$

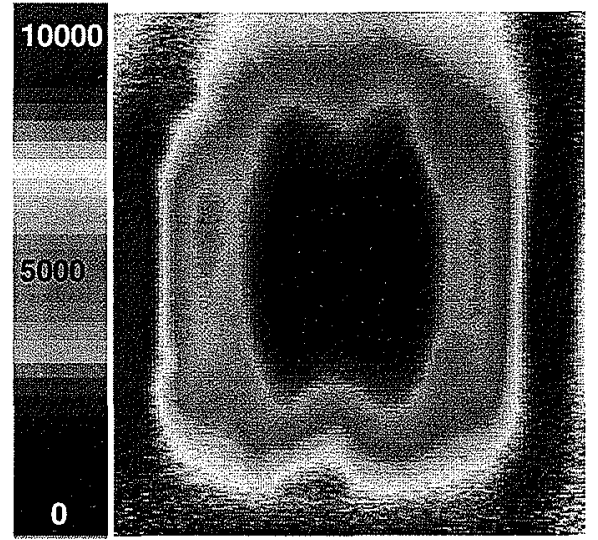

Intensity $(\mathrm{V} / \mathrm{m})^{2}$

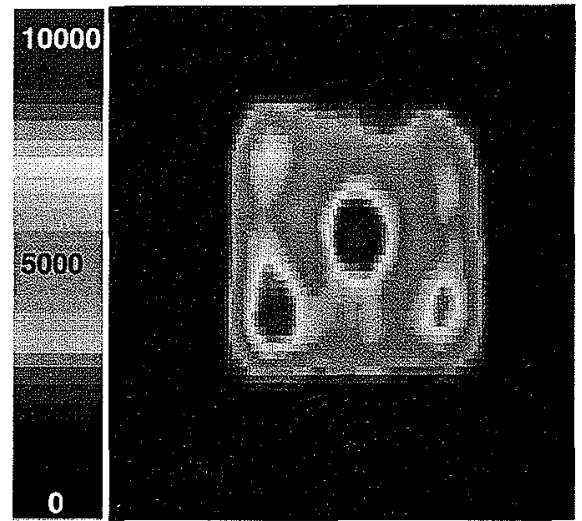

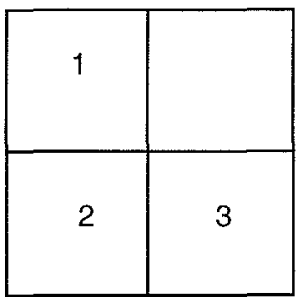

1 : Intensity from transmission measurement

2 : Intensity from interferometry

$3:$ Phase from interferometry

Fig. 7 - NDE of the highly absorbing sample of fig. 2 (left). Amplitude modulation and lock-in detection at 1 $\mathrm{Hz}$ with an Agema 782. Comparison between transmission and interferometric methods.
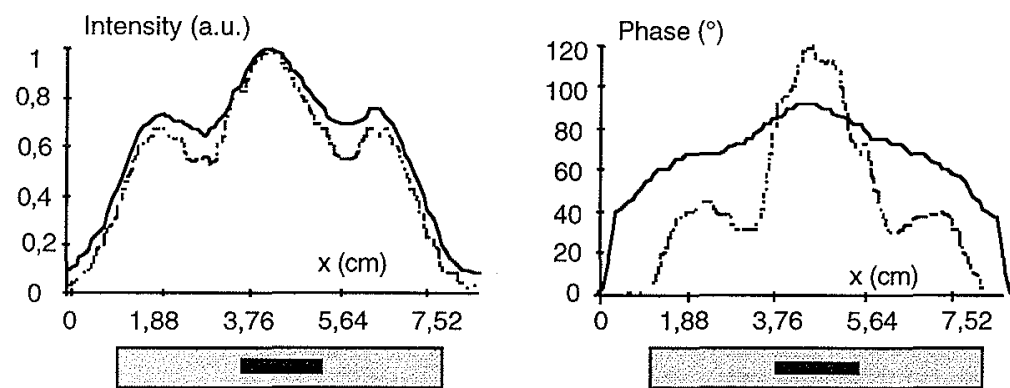

Fig. 8 - NDE of the absorbing sample (fig. 2 - left) by interferometric method. Comparison between steady state and amplitude modulated $(0.75 \mathrm{~Hz})$ measurements. Horizontal distributions of amplitude (right) and phase (left). _ _ : steady-state; - . - : amplitude modulation (from interferometric images of fig. 7). 